\title{
Contributions on Laser Driven Inertial Confinement Fusion
}

\author{
Heinrich Hora \\ Department of Theoretical Physics, \\ University of New South Wales, Sydney 2052, Australia
}

\begin{abstract}
The following modified preprint of a chapter in the forthcoming book by Guillermo Velarde and Natividad Carpintero Santamaria Inertial Confinement Nuclear Fusion: A Historical Approach by its Pioneers with personal comments is presented here as an example about the long years difficult developments towards the aim for producing unlimited, safe and clean nuclear energy in the same way as it is the energy source of the sun. There are arguments that the most recent developments with the plasma block ignition using petawatt-picosecond laser pulses may lead to a fusion power station with a highly simplified operation such that the cost of electricity may be three or more times lower than any energy source on earth, opening the golden age with dramatic consequences for human life and the environment. Applied sciences in all fields, economics and politics may be stimulated just by considering these consequences though these new results on Inertial Fusion Energy (IFE) need to be further examined and developed on a broad basis.
\end{abstract}

Key words: Fusion energy, Laser Fusion, Inertial confinement fusion, Volume ignition, Skin layer driven plasma blocks, Nonlinear ponderomotive force, Igniton of uncompressed solid DT, Relativistic serf-focusing, Biographic review

\section{INTRODUCTION}

Lasers open the way to fusion energy by inertial confinement: After having learnt computer programming with stone age machine codes up to the Fortran at IBM and leaving behind some early success on radiation emission from solids and on semiconductor physics $^{[1]}$, I joined the Institute for Plasma Physics (later Max-Planck-Institute) in Garching/Germany in October 1962 with the aim to study laser-plasma interaction for inertial confinement fusion using an IBM 7040 machine. First I had to learn plasma physics where I showed how the complex optical constants for Schlüter's two-fluid equations could be derived with Spitzer's collision frequency arriving at the basically same values as the derivations from quantum electrodynamics (Gaunt) used in astronomy and same values from the inverse bremsstrahlung theory. To treat laser-plasma interaction I could use my preceding work on electromagnetic wave propagation in inhomogeneous media where I used exact solutions with elementary functions for Rayleigh profiles and more generally the WKB approximation.

With these tools I could approach the laser-plasma interaction theory for fusion applications, however, I saw from primitive fusion estimations what rather confusing predictions on fusion resulted. Having witnessed the lecture by Basov and Krokhin at the UNESCO center in Paris at the Quantum Electronics Conference February 1963 and having seen the Westinghouse report by Albert Engelhardt very similar to the latter seen reproduction of the Basov-Krokhin paper by John Dawson - well appreciating some numerical extension by Dawson - I used their self similarity model for the hydrodynamics and my results on optical plasma properties to perform systematic laser fusion computations for adiabatic expanding plasmas. The input was a uniform initial maximum plasma density $\mathrm{n}_{\mathrm{o}}$ expressed in multiples of the solid state density $n_{s}$ where the spherical plasma had received an energy $E_{0}$ expressed in relation to a break-even energy $\mathrm{E}_{\mathrm{BE}}$. Using the well known fusion cross sections for DT (Deuterium and Tritiuim), I found an agreement with the few cases calculated by Basov and by Dawson. These values showed very low gains of fusion energy, but by systematically computing cases with varying parameters I found the best optimum values published in my Institute's Report on July 1964 (translated in Canada in $1965^{[2]}$ ). Fig. 1, My computations for various densities and energies resulted in the plots with the parabolic curves in the mentioned IPP-report arriving at the highest gains $\mathrm{G}$ according to:

$\mathrm{G}=\left(\mathrm{E}_{\mathrm{o}} / \mathrm{E}_{\mathrm{BE}}\right)^{1 / 3}\left(\mathrm{n}_{\mathrm{o}} / \mathrm{n}_{\mathrm{s}}\right)^{2 / 3}$

The optimum DT plasma temperature was found to be $17 \mathrm{keV}$ (about 197 Million degrees Kelvin).

What was downcasting and disappointing was that the break-even energy $\mathrm{E}_{\mathrm{BE}}$ was about $6 \mathrm{MJ}$ (mega joule) while the best Q-switched lasers at this time had pulses of few Joules for a few ns (nanosecond) duration. On top the gain increased on the cubic root of the input 
energy $\mathrm{E}_{\mathrm{o}}$ only. But what was evident from the formula (1) was that an increase of the initial density e.g. to 1000 times the solid state needed than one million times less energy $E_{o}$ to reach the same gain. Therefore compression was the key word which was well known from uncontrolled fusion reactions.

This gain formula (1) is algebraically identical [ ${ }^{[3]}$, Eq. (13.8)] with the formula $G=$ const $\times n_{0} R$ (where $R$ is the radius of the spherical plasma compressed to the initial density $\mathrm{n}_{\mathrm{o}}$ ) which was published by Ray Kidder.

In 1974 after my formula had been published in several places before, e.g. at the Quantum Electronics Conference in Osaka 1970. I even was the referee for Kidders publication in Nuclear Fusion but I was too tired to insist "that the author should first confirm that his consort. Agrees with my earlier values". Indeed I had checked that there was an agreement within a factor too and recommended publication. Later, my first book about laser fusion 1975 was knocked down by Moshe Lubin with the argument that I did not use Kidder's formula but only my algebraically identical however much more instructive formula (1). Indeed the use of compression was fully familiar with John Nuckolls at Livermore from his classified work which partially was declassified by the famous lecture 1972 of Edward Teller at the Quantum Electronics Conference in Monteral $^{[4]}$. Nuckolls had most pioneering results since 1960 and showed his unquestionably correct gains at 10,000 times solid state density in $1972^{[5]}$ what was fully wrongly criticized by others later.

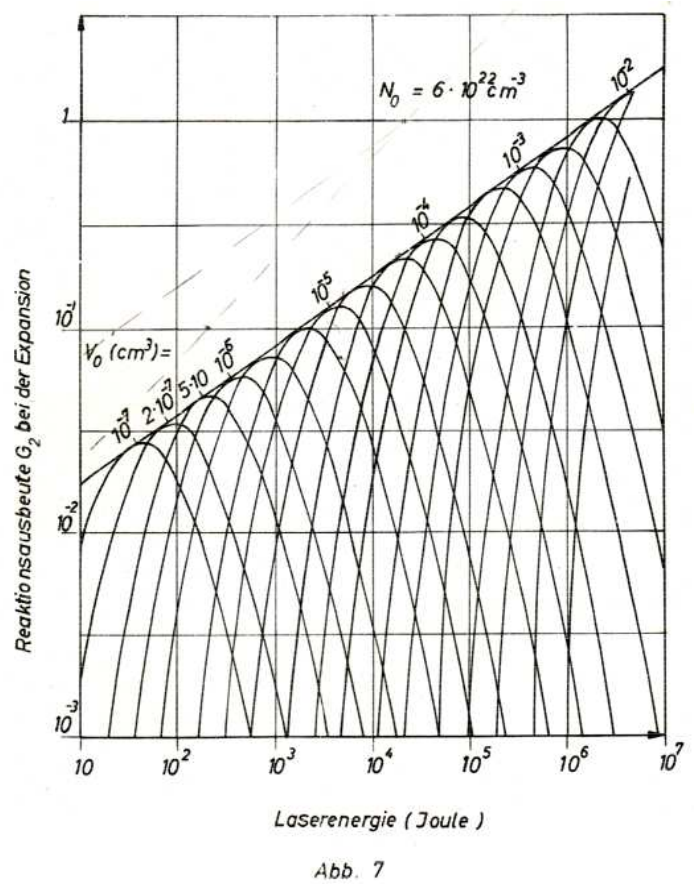

Fig. 1: DT fusion reaction gain per energy $E_{o}$ input into the sphere of volume $\mathrm{V}_{\mathrm{o}}$ (radius $\mathrm{R}$ ) at a solid state density $\mathrm{n}_{\mathrm{o}}=\mathrm{N}_{\mathrm{o}}=6 \times 10^{22} \mathrm{~g} / \mathrm{cm}^{3}$ depending on the laser energy Eq. 1.
These computations seemed to be rather convincing and without difficulties if there was not the question, how to get the energy $\mathrm{E}_{\mathrm{o}}$ uniformly into the compressed plasma sphere. When performing the first laser-plasma experiments after the clean Q-switch laser pulses were available in 1962 through Hellwarth at the Hughes Aircraft Laboratory in Malibu, a crucial new area in physics was opened: nonlinear physics ${ }^{[6]}$. Before, the laser pulses of up to $1 \mathrm{MW}$ power irradiating targets showed fully classical interaction. The targets were heated to temperatures of about $30,000 \mathrm{~K}$ and the emitted ions had the expected energies of up to few $\mathrm{eV}$ (electron volts). When the Linlor next door to Hellwarth irradiated with $10 \mathrm{MW}$, suddenly he measured huge amounts of highly charged ions of up to $10 \mathrm{keV}$ energy ${ }^{[7]}$ which just is necessary for nuclear fusion of DT. Linlor was immediately promoted to the Atomic Energy Commission in Washington to work on laser fusion.

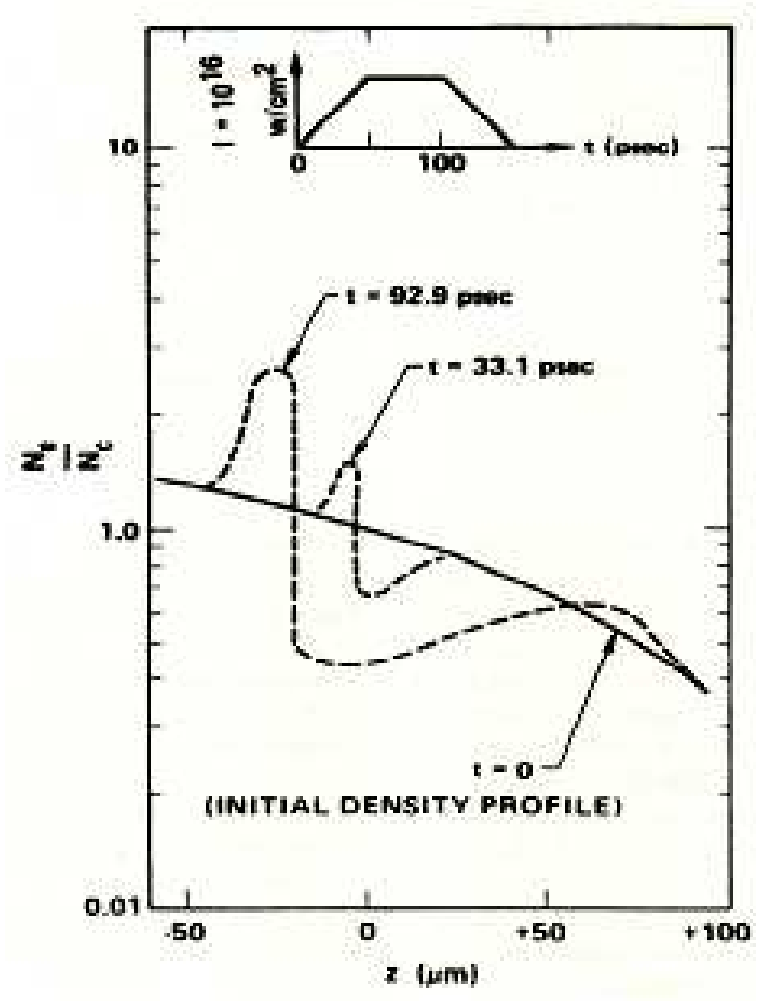

Fig. 2: A neodymium glass laser pulse (upper part) irradiates a deuterium plasma with initial density $n_{e} / n_{c}\left(n_{c}\right.$ is the critical density of $10^{21}$ ions $/ \mathrm{cm}^{3}$ ) where the whole plasma dynamics is dominated by the nonlinear force ${ }^{[9]}$ and results in the dashed density at times $t$ after the beginning of the laser pulse (Shearer, Kidder and Zink, LLNL 1969, see ${ }^{[3]}$, Fig. 10.7] 
But it turned out that there was not the expected temperature of above 100Million degrees Kelvin, the plasma still remained at very much lower temperatures and it was just a new kind of ion acceleration due to unknown processes which were shown to be essentially nonlinear and not of thermal nature. Characteristic of this was that the large number of ions (much larger than ambipolar thermal processes could explain) was separated linearly by their energy on their ion charge number Z [3, Fig.1.9], what therefore was typically for a non-thermal process.

New nonlinear effects of laser-plasma interactions: In order to follow this up, I looked into the optics properties of laser-plasma interaction, especially what the variation of the refractive index in the inhomogeneous plasma corona does with respect to electrodynamic interaction forces. Indeed a force of this kind was known as electrostriction by Kelvin since 1846 reformulated later by Helmholtz as "ponderomotive" force in electrostatics. For high frequency fields, Erich Weibel was the first to see formally the same forces proportional to the negative gradient of the electric field vector $\mathrm{E}$ given by a force density:

$\mathrm{f}_{\mathrm{NL}} \propto-\nabla \mathrm{E}^{2}$

For the dielectric properties of plasmas, I found the first approach using the fields in inhomogeneous plasmas $^{[8]}$.

At this time, Ray Kidder in Livermore led a very interesting experiment with spherical irradiation of 16 laser beams on a spherical target. From this group a paper by Gregg and Thomas was published about the usual observation of the $\mathrm{keV}$ ions, but Kidder tried to see the spherical compression. Instead, the sphere produced plasmas moving each like spikes against each laser beam such that the plasma looked like the fruit of a chestnut. I was there end of 1966 of an APS conference in Stanford and was led to the Livermore experiment. I had to pass three gates with barbed wire and my photo and the fingerprints were checked. Kidder came and asked me a number of questions about my work. When I tried to ask a question, I was told this cannot be done.

By the way, following my results in the IPP 6/23 report I submitted a patent with Benedikt Kronast on laser fusion. This patent was classified and I had, as a German citizen, to sign to be quiet or to be punished up to the electric chair. Later the patent was declassified and granted in the USA (3444377) and other countries.

In order to understand the strange laser-plasma interaction, I could first conclude the linear Zdependence of the acceleration of the very energetic ions and could suggest how the nonlinear force dielectrically was getting the plasma corona to a nonthermokinetic (low temperature) explosion into a part of plasma moving against the laser light and another plasma block moving into the plasma interior with the $\mathrm{keV}$ (and later $\mathrm{MeV}$ and $\mathrm{GeV}$ ) ion energies ${ }^{[9]}$. I was then invited to join the experiments of Engelhardt at Westinghouse where I could in all details quantitatively analyze their excellent experimental results by ordering the numerous parameters to see the gas dynamic interaction with clear spherical plasma expansion and the energetic nonlinear force driven plasmas. This was published in the first volume of Laser Interaction and Related Plasma Phenomena (LIRPP1, p. 273) of which Plenum Press sold more than 1500 copies.

My appointment at Westinghouse was tenured as a Senior Physicst with all privileges of pension, insurance etc.. The laser fusion project was then stopped due to the financial problems of the US with the Vietnam war. I could have continued at Westinghouse on a project on which I had published on defect generation in solids. Further I got offered a deputy director's position at a Fraunhofer Institute in Freiburg/Germany on semiconductor physics and an offer as Associate Professor at Rensselaer Polytechnic Institute but I followed up my position at Garching from where I was on leave and could continue on laser driven ICF. I could work part time at the position at Rensselaer is found there the conference series "Laser Interaction and Related and Related Plasma Phenomena" apart from other research (Schwarz-Hora effect; Goos-Haenchen effect for Schrödinger waves with the phase versus intensity problem of quantum mechanics etc.).

A problem arose for me in the Garching end of 1969 with the politics of Willy Brandt in connection with the non-proliferation treaty when my statements on laser fusion supported the arguments of the opposition of Dr. Franz Josef Strauss which was backed by the governments of Switzerland, South Africa (Dr. Roux) and others. My difficulties with the red court Camarilla of Brandt (which machinations appeared to be more servile and oppressive than the court Camarilla of Kaiser Wilhelm) let me finally to accept the foundation chair of theoretical physics at the University of New South Wales in Sydney/Australia to establish a new department and where I with several research students, guests and some associates in my department could work on laser fusion with good outside support.

An essential progress of my formulation of the dielectric explosion of the nonlinear laser-plasma interaction mechanism developed in 1969 at Livermore with the thesis of J.W. Zink supervised by James Shearer within the project of Kidder. Numerically it was shown how the nonlinear force of a $10^{16} \mathrm{~W} / \mathrm{cm}^{2}$ neodymium glass laser intensity irradiating an initially linearly increasing ramp of plasma, is creating the fast plasma block moving against the laser light and how a density minimum is generated near the critical density, Fig. 2, which later was called a caviton together with a steepening of the density profile to the plasma interior. This important numerical discovery was then the 
convincing tool to measure the cavity as proof of the action of the nonlinear force. This succeeded so splendidly with microwaves by Wong and Stenzel and then with lasers by Zakharenkov et al., by Azechi et al. [10, Fig. 4-11] and many other groups.

Another experimental confirmation of the nonlinear force was the measurement of the electrons emitted laterally from a laser beam focused in tenuous helium at $10^{16} \mathrm{~W} / \mathrm{cm}^{2}$ neodymium glass laser irradiation resulting in an electron energy of about one $\mathrm{keV}$ corresponding exactly to the value expected from the nonlinear force.

The polarization independence agreed also with the result $-\nabla \mathrm{E}^{2}$ for the force. When one, however, was trying to see how the quiver motion was converted into translating motion, the energy of $\mathrm{keV}$ was reproduced only into the E-direction of the laser field and not any acceleration was along the $\mathrm{H}$-direction if the laser field was presented as being fully transversal by cutting a Gaussian profile from a plane wave field. This led to recognize that this laser field was not Maxwellian exact and that it had surprisingly a very little longitudinal component to be exact. With this tiny addition, the computation of the electron motion along $\mathrm{H}$ changed from zero to the $\mathrm{keV}$. This led to realize the nonlinearity principle: the neglect of very minor linear physics parts can change a result from wrong to right, from yes to no! Nonlinear phenomena are therefore not a higher order extension only with some percentage corrections of results, but can totally confuse results. We knew in what direction we had to look and solved the problem $\mathrm{PhD}$ Thesis of L. Cicchitelli ${ }^{[12]}$ with a better understanding of laser beams ( $\mathrm{PhD}$ of $\mathrm{R}$. Castillo). Otherwise realizing this principle opens the possibility that in future we can systematically derive the phenomena of which nobody could have dreamed of with fundamentally new applications in life and society. We have then to explore linear physics to even higher accuracy and expand mathematics and computations enormously for systematically exploring this nonlinear physics. A basically new dimension of physics is then opened. This is in contrast to the expectation of saturation of physics knowledge and the end of physics concluded by Steven Hawkings or Carl Friedrich von Weizsäcker ${ }^{[10]}$.

The nonlinear force of laser-plasma interactions: The general derivation of the nonlinear force ${ }^{[9]}$ from my Westinghouse time led to the discovery of two extra nonlinear terms additional to the one which Schlüter had derived in his space charge neutral two-fluid hydrodynamic equations. Indeed he had derived (in contrast to Spitzer) the other nonlinear term which achievement was extremely difficult but he mastered it. For me, the derivation was not so difficult since I knew from momentum conservation at laser-plasma interaction, what was missing. The only printed derivation of the so completed space charge neutral hydrodynamic equations are only in my books ${ }^{[3,10]}$ and a textbook on electrodynamics ${ }^{[13]}$. Schlüter mentioned recently that he still does not believe in my result.

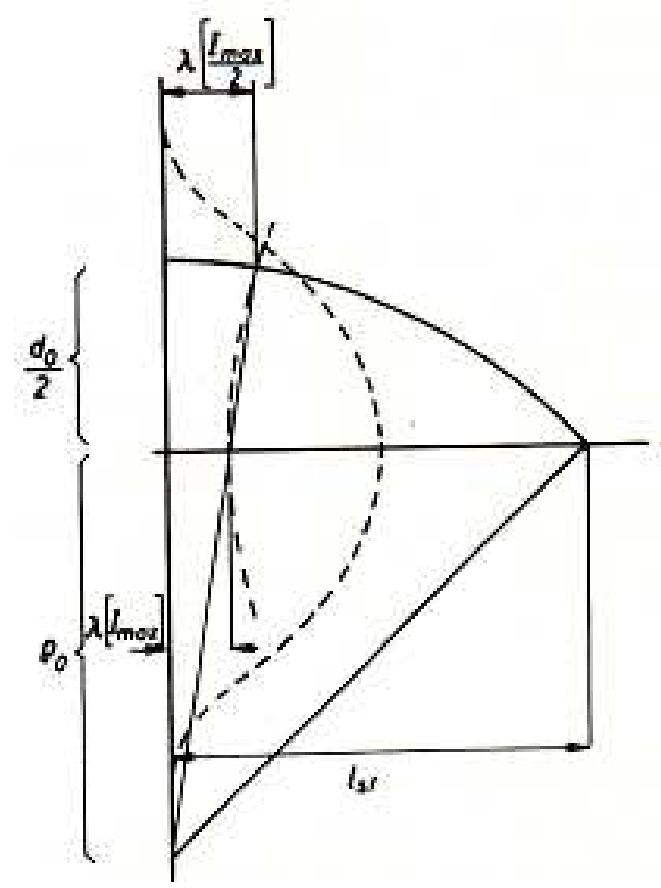

Fig. 3: Scheme for relativistic self-focusing ${ }^{[17]}$. From the left in a vacuum, a laser beam with a kind of Gaussian intensity profile (dashed) is incident on a uniform plasma. The plane wave front moves into the other dashed line since the effective wavelength at higher intensity is shorter than al lower intensities due to relativistic mass change of the quivering electrons. [By Bruce Boreham ${ }^{[11]}$. The maximum electron]

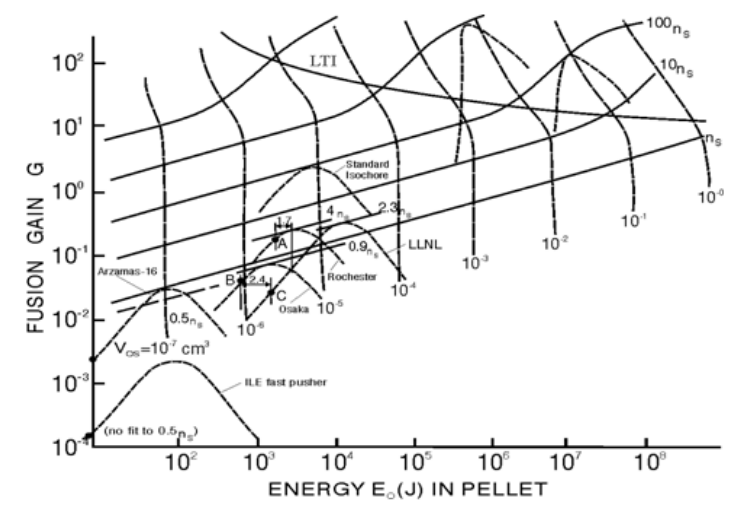

Fig. 4: Fusion gains for DT similar to Fig. 1 with very similar parabolas in the lower left part which for higher gains than 8 are deformed due to volume ignition $^{[22]}$ by self-heat from reaction products and by re-absorption of bremsstrahlung. The lines are for the optimum gains in multiples of the solid state density $n_{s}$ at maximum compression. The measured values from direct drive of different laboratories fit the calculations and confirm that adiabatic self similarity compression. 
For the time dependent nonlinear force, the first theory came from Klima and Petrzilka ${ }^{[14]}$ in agreement with my formulation for plane geometry. For the general case a hefty controversy arouses with six groups having six different formulas including ter-Haar, Washimi, Karpman, Tskhakaya, Kono and others. With a rather unusual polemic interpretation of this confusing situation, it was nearly solved by the work of Zeidler, Mulser and Schnabl, but only "nearly". A very small logarithmic term was still missing which I found from algebraic symmetry ${ }^{[15]}$. The final completeness of this and only this formulation of the nonlinear force was proved in a Ph.D. Thesis by Rowlands (1990) from Lorentz and gauge invariance.

After this all I am summarizing the final general formulation of the nonlinear force as the nonthermokinetic part $\mathbf{f}_{\mathrm{th}}$ of the general force density in a plasma (i.e. after subtraction of $\mathbf{f}_{\mathrm{th}}=-\nabla \mathrm{p}$ with the gas dynamic pressure p) as the electrodynamic field produced nonlinear force density ${ }^{[3,9,10,15]}$ :

$$
\begin{aligned}
& \mathbf{f}_{\mathrm{NL}}=\nabla \bullet\left[\mathbf{E E}+\mathbf{H H}-0.5\left(\mathbf{E}^{2}+\mathbf{H}^{2}\right) \mathbf{1}+(1+(\partial / \partial \mathrm{t}) / \omega)\right. \\
& \left.\left(\mathbf{n}^{2}-1\right) \mathbf{E} \mathbf{E}\right] /(4 \pi)-(\partial / \partial \mathrm{t}) \mathbf{E} \times \mathbf{H} /(4 \pi \mathrm{c})
\end{aligned}
$$

where, $\mathbf{1}$ is the unity tensor, $\omega$ the laser frequency and $\mathbf{n}$ is the (complex) refractive index. This can be reformulated by tensor algebra into:

$$
\begin{aligned}
& \mathbf{f}_{\mathrm{NL}}=\mathbf{j} \times \mathbf{H} / \mathrm{c}+\mathbf{E} \rho+\mathbf{P} \bullet \nabla \mathbf{E} / 4 \pi+(1 / \omega)(\partial / \partial \mathrm{t}) \mathbf{E} \nabla \bullet \\
& \left(\mathrm{n}^{2}-1\right) \mathbf{E} / 4 \pi+\left[1+(1 / \omega)(\partial / \partial \mathrm{t}]\left(\mathbf{n}^{2}-1\right) \mathbf{E} \bullet \nabla \mathbf{E} / 4 \pi\right.
\end{aligned}
$$

It was shown ${ }^{[9,15]}$ that these algebraically identical formulations are Lorentz and gauge invariant and are the complete description of the force density in the plasma due to electric and magnetic fields. The formulation (3) is that of the Maxwellian stress tensor including the dielectric response and transient (time dependent) behavior of the fields. The formulation (4) explains the parts acting in the nonlinear force. Here one recognizes on the right hand side first the Lorentz term $\mathrm{f}_{\text {Lorentz }}=\mathrm{j} \times \mathrm{H} / \mathrm{c}$ to the plasma current density $\mathrm{j}$ and the vacuum velocity of light $\mathrm{c}$, then the Coulomb term E $\rho$ with the electric charge density $\rho$ and as the third term the Kelvin ponderomotive term $\left({ }^{10]}\right.$ see Eq. 1):

$$
\begin{aligned}
& \mathbf{f}_{\text {Kelvin }}=\mathbf{P} \bullet \nabla \mathbf{E} / 4 \pi=\left(n^{2}-1\right) \nabla \mathbf{E}^{2} / 8 \pi- \\
& \left(\mathbf{n}^{2}-1\right) \mathbf{E} \times(\nabla \times \mathbf{E}) / 4 \pi
\end{aligned}
$$

The remaining terms in Eq. 4 are new nonlinear terms which were derived from the general equation of motion in plasmas from the studies of laser interaction $^{[10]}$. The proof for the final generality of Eq. 4 was given by momentum conservation ${ }^{[9]}$ for the nontransient case $(\partial / \partial t=0)$ and for the transient case of symmetry methods ${ }^{[15]}$.
For the correct interpretation it is necessary to mention that Kelvin's ponderomotive force is identical with the nonlinear Schlüter term ${ }^{[10]}$ :

$$
\mathbf{j} \bullet \nabla\left(1 / \mathrm{n}_{\mathrm{e}}\right) \mathbf{j m} / \mathrm{e}^{2}=\left(\omega_{\mathrm{p}}{ }^{2} /\left(\omega^{2}\right) \mathbf{E} \bullet \nabla \mathbf{E} / 4 \pi\right.
$$

Remembering the definition of the electric polarization $\mathrm{P}$ and the refractive index without collisions:

$\mathbf{P}=\left(\mathbf{n}^{2}-1\right) \mathbf{E} / 4 \pi$

From Kelvin's ponderomotive force (5) follows formally an expression of the "field gradient force" or the electrostriction for collisionless plasma ( $\mathrm{n}$ without imaginary part):

$\mathbf{f}_{\mathrm{NL}}=\left(\mathbf{n}^{2}-1\right) \nabla \mathbf{E}^{2} /(8 \pi)$

This can be used in the case of perpendicular incidence of plane laser waves on an inhomogeneous plasma of one dimensional geometry e.g. along the coordinate $\mathrm{x}$. For the same conditions, the stress tensor description produces a force density in the $\mathrm{x}$-direction of:

$\mathbf{f}_{\mathrm{NL}}=-(\partial / \partial \mathrm{x})\left(\mathbf{E}^{2}+\mathbf{H}^{2}\right) /(8 \pi)$

Formulation (8) led to the common expression of the ponderomotive force for (8). As is known for (plane wave) perpendicular incidence of laser radiation on a plasma, the Schlüter term is then zero. Nevertheless there is a force of the form of Eq. 5. In this case, however, the nonlinear force $\mathrm{f}_{\mathrm{NL}}$ is the result of the Lorentz term in Eq. 4. This confusion of the definitions is avoided if one uses the general expression of the nonlinear force (3) for the electrodynamic part of the force density in a plasma. This is valid for any incidence, for plasma with collisions and including time dependence of the fields.

With the results ${ }^{[9]} \mathrm{I}$ derived the first theory of ponderomotive self-focusing ${ }^{[16]}$ showing why the laserplasma interaction changes at MW laser intensities from the classical gas dynamics into the nonlinear physics range. My general theory of the relativistic selffocusing ${ }^{[17]}$, Fig. 3, - expanded by the PhD work of E.L. Kane ${ }^{[18]}$ and the $\mathrm{PhD}$ at the University of Giessen of $\mathrm{T}$. Häuser ${ }^{[19]}$ - could explain why the laser beam shrinks relative to the half wavelength diameter and how then the very high beam intensity accelerated Zseparated ions to energies above $\mathrm{MeV}$ exactly as measured from laser produced plasmas (Ehler, LutherDavies), ${ }^{[3]}$, Sect. 12.2\&12.6]. Another triumph of the nonlinear force was when Francis Chen in $1974^{[20]}$ could derive in a very general way that the numerous parametric instabilities at laser-plasma interaction were all due to the nonlinear force. My result of 1969 
contained also the momentum flux density of the electromagnetic energy in the inhomogeneous plasma showing this was half of the Abraham and half of the Minkowski formulation. This led to the solution of the Abraham-Minkowski problem at least four fully ionized plasma (PhD of M.M. Novak) [ ${ }^{[3]}$, Sect.9.4] apart from a basic aspect of quantization: if action is less than $h$ there is no energy conservation ( $\mathrm{PhD}$ of $\mathrm{R}$. Sutherland).

Discovery of volume ignition: Back to my computations of fusion gains, it was evident how poor our formula (1) and the equivalent later one of Kidder was. No fuel depletion, no self-heat from the reaction products and no re-absorption of the bremsstrahlung in the reacting plasma was included what we all then followed up. An essential ingredient was the inclusion of a collective stopping power theory with which we could immediately explain the measured (Kerns et al., Kirtland Air Force Base) very anomalous short stopping length of relativistic electrons in plasmas. We learnt later that this collective model was derived before from Denis Gabor ${ }^{[21]}$ in contact with the pioneer of plasma theory, S.R. Milner who before Debye had derived the Debye length. Our work (PhD thesis of $\mathrm{P}$. S. Ray 1977) led to the discovery of the volume ignition of a laser heated and compressed spherical plasma $^{[22]}$. This result was confirmed in 1981 by $\mathrm{R}$. Kirkpatrick and John Wheeler ${ }^{[23]}$. We showed that nearly the same high fusion gains could be achieved by the "robust" volume ignition, as Colgate, Lackner et al. Underlined $^{[24]}$ in contrast to the hope of fashionable central spark ignition with its very non-natural temperature and density profiles and difficulties with symmetry and instabilities. Very sophisticated extensions and improvements of the volume ignition by Martinez-Val, Eliezer, and Piera and by Xiantu He and Y.-S. Li should be highlighted. After McCrory publicly ridiculed my initial computations by claiming that I were cheating by using wrong constants, we published an extended version of the code (Stening et al., LIRPP10, 1992) such that everyone can convince him (her) self what was calculated.

One triumph is that the highest ever published direct drive fusion gains from Osaka, Livermore, Arzamas-16 and Rochester fully agree with our shockand stagnation-free volume compression computations $^{[25]}$ which were confirmed empirically as "Yamanaka compression". It should be mentioned that Mike Campbell - familiar like nobody else with nearly all details of laser technology and spark ignition, however influenced by his theoreticians - commented Dec. 2004, that the mentioned agreement may be due to the insufficient quality of the laser pulses at Osaka and Rochester. Nevertheless the mentioned Livermore (Storm et al., 1986) direct drive result was an ideally fitting volume burn in the same perfect way as the others. This is in contrast to fast pushers where their strong shock generation prevented high gains.
Hydrodynamic computations and the genuine twofluid model: Hydrodynamic codes were developed to study details of the nonlinear force interaction in plane geometry following the mentioned success by Shearer Kidder and Zink with the WAZER code from early 1970, Fig. 2. Rick Kinsinger at Rochester developed a very detailed code where the exact electromagnetic wave field in the plasma corona was used on top of a one-fluid differentiation of electron and ion temperature and mutual coupling description. I had to add only nonlinear generalizations of the optical constants and as main part - the inclusion of the nonlinear force. The result of irradiation of deuterium with initially linearly increasing density along 50 wave length before critical by up to $10^{18} \mathrm{~W} / \mathrm{cm}^{2}$ laser pulses - mostly done later in a $\mathrm{PhD}$ thesis by Vincent Lawrence - showed a very depressing result. The light was initially reflected at the critical density (mirror reflection) with a low net reflection due to absorption along the plasma corona. The partially reflected light field caused standing waves through which the hydro-motion of the plasma was too slow to pass such that the nonlinear force produced a density ripple within few ps and then a high von-LaueBragg reflection appeared as a phase reflection at the very low peripheral densities prohibiting the laser light to deposit its energy into the plasma. The plasma did all to prevent laser energy absorption by heating or nonlinear force dynamics: Moshe Lubin was very depressed.

With this very sophisticated hydrocode with one dimension geometry we avoided the density ripple generation with low reflectivity Rayleigh density profiles in the beginning resulting later in the before mentioned complications. One result is shown in Fig. 5.

A neodymium glass laser intensity of $10^{18} \mathrm{~W} / \mathrm{cm}^{2}$ produced a deuterium block of more than 15 wave length thickness moving with about $10^{9} \mathrm{~cm} / \mathrm{Sec}$ speed against the laser light after $1.5 \mathrm{ps}$ interaction and a similar block was moving into the plasma interior. Laser pulses of such intensities and of such short duration, however were not available at 1980, the time of computations.

Fortunately the WAZER code at Livermore was rather primitive and did not include the whole wave field with the reflection, otherwise Shearer, Kidder and Zink would not so easily have discovered the caviton. The change between mirror and phase reflection could be seen (Lubin 1974 Phys. Rev. to be published which never was printed!) from the stochastic change of the measured net reflection between few percent and longer periods of 10 and more ps of high reflection. We considered to prepare a patent with phase modulation in a ps scale, but this did not proceed. Maybe that this was in the air around 1980 when Kato was in Rochester and where his discovery of the random phase plate was introduced to suppress ponderomotive self-focusing and filamentation. 


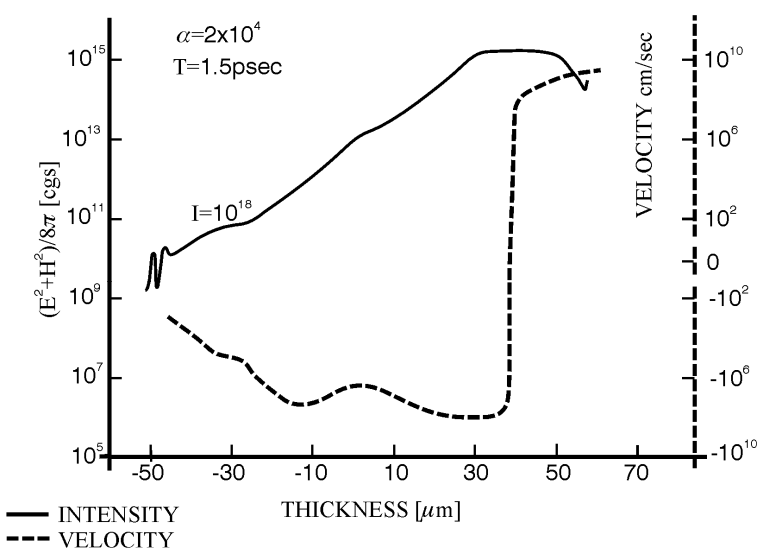

Fig. 5: Generation of blocks of deuterium plasma moving against the neodymium glass laser light (positive velocity $\mathrm{v}$ to the right) and moving into the plasma interior (negative velocities) at irradiation by a neodymium glass laser of $10^{18} \mathrm{~W} / \mathrm{cm}^{2}$ intensities onto an initially $100 \mathrm{EV}$ hot and $100 \mu \mathrm{m}$ thick bi-Rayleigh profile (Fig. 10..17 of $^{[3]}$ ) with minimum internal reflection. The electromagnetic energy density $\left(\mathrm{E}^{2}+\mathrm{H}^{2}\right) /(8 \pi)$ for calculating the nonlinear force, Eq. (9) is shown at the same time of $1.5 \mathrm{ps}$ after begin of the constant irradiation $^{[9]}$.

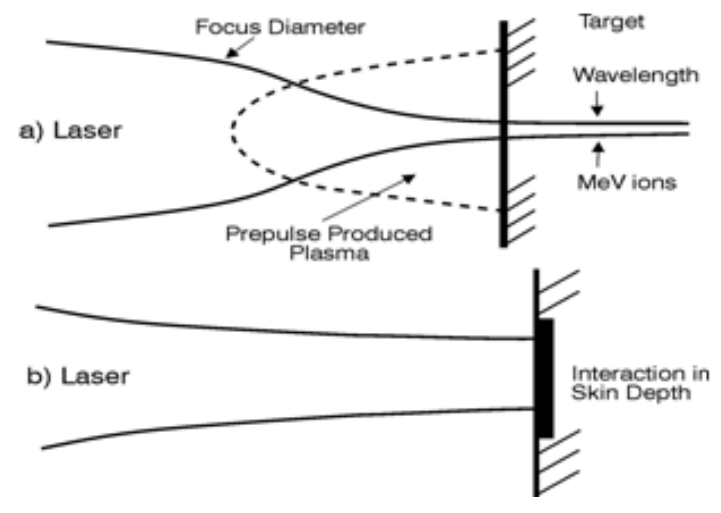

Fig. 6: Relativistic self-focusing squeezing the laser beam down to about wave length diameter due to the relativistic plasma response in the prepulse produced plasma (a). If relativistic self focusing is not happening, the laser beam interaction in the skin layer of the target surface as the plane geometry case (b) ${ }^{[40]}$

Finally it turned out that this beam smoothing was - as expected - eliminating the density ripple and the finally it turned out that this beam smoothing was - as expected - eliminating the density ripple and the phase reflection in order to get high laser energy input into the plasma ${ }^{[26]}$. Indeed the stochastic pulsating interaction on the 10 ps scale was measured independently from this all by Luther-Davies and Maddever ${ }^{[27]}$, to which the mention ripple suppression was the numerical confirmation when using broadband laser spectra ${ }^{[26]}$. This is similar to random phase plates ${ }^{[28]}$ where against the initial expectation by C. Labaune et al ( $\mathrm{see}^{[28]}$ ) to suppress self-focusing but showed the suppression of the pulsation. This permits then to use red laser light with appropriate smoothing for direct drive instead of using expensive higher harmonic generation. This suppression of ripples also suppresses parametric instabilities by a factor 100 (!!) as confirmed experimentally by Guiletti et al. (LIRPP9, p. 261) leading to an increase of direct driven fusion gains by a factor $50^{[29]}$.

For plane wave interaction we developed a genuine two-fluid code where the electrical coupling between the electrons and ions was included $(\mathrm{PhD}$ of P. Lalousis). This gave revealing insights for the coupling of the laser field with the longitudinal plasma oscillation and the generation of quasiLangmuir waves (due to the inhomogeneities no longer waves, only quasi-waves) showing all details of electric double layers numeric, even the inverse double layers at cavitons ${ }^{[30]}$. More analytical results could be derived generalizing ambipolar phenomena with nonlinear terms, e.g. a new resonance at perpendicular incidence and long wavelength structuring at the low density corona (PhD. M. Goldsworthy $)^{[31,32]}$ as observed ( $\mathrm{Gu}$ Min and Aleksandrova, Brunner, Sklizkov et al.) and being suppressed following our theory.

The double layer theory led to the derivation of surface tension of plasma, stabilization of surface waves at short wave length, explaining why the measured Rayleigh-Taylor instability is less dangerous than all established theories claim. The generalization to the degenerate electron gas (R.S. Pease et al. $)^{[33]}$ arrived at a direct quantum theory of surface tension of metals and generalizing this to the Fermi-Dirac energy of nucleons leads to a new theory of nuclear forces based on a kind of Debye lengths ${ }^{[34]}$ explaining why endothermic nuclear synthesis is possible under equilibrium only to about uranium and why at six times higher than nuclear density the then relativistic branch of the Fermi energy of nucleons prohibits nucleation and the quark-gluon soup is produced. It also explains the jump in Bagge's series for the magic nuclear numbers without needing the Jensen \& Goeppert-Mayer solution. And this all is a consequence of the ugly and only a low level classically degraded plasma physics! 


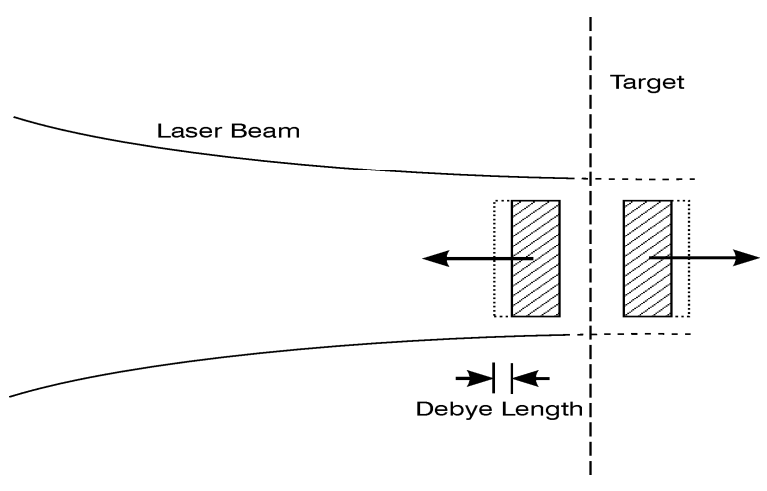

Fig. 7: Scheme of skin depth laser interaction where the non-linear force accelerates a plasma block against the laser light and another block towards the target interior. In front of the blocks are electron clouds of the thickness of the effective Debye lengths of less than 500 $\mathrm{nm}^{[44]}$. The compressing block with $10^{111} \mathrm{~A} / \mathrm{cm}^{2}$ DT ion current density would be sufficient to ignite a fusion reaction wave in uncompressed solid DT.

Campbell's initiation of the fast ignitor: When Azechi et al. in $1991^{[29]}$ measured laser compression of polyethylene by lasers to 2000 times the solid state density, an unexpected low temperature of $300 \mathrm{eV}$ (3.48 Million degree Kelvin) only was measured against expected much higher value. Hopes for laser fusion were disappointed. It was Mike Campbell who had the idea $^{[35]}$ that the just then discovered a CPA technique to produce ps-PW laser pulses could be used to add up the necessary heating after the enormous compression which scheme as fast ignitor was then developed in cooperation with Max Tabak and others.

Still as an alternative to fast ignition and using lower temperatures resulting from Azechi et al., it was possible that we recalled our volume ignition computations where at sufficiently large laser pulse energies and sufficient high compression, the ignition temperature could well be near $500 \mathrm{eV}$ and even lower. One result is that if compression could be increased from 2000 to 5000 times the solid DT and if a red smoothed neodymium glass laser pulse of $10 \mathrm{MJ}$ (twice that of NIF) is incident that the gang of 36 (fusion produced electric energy per incident laser energy) can be achieved by volume ignition ${ }^{[36]}$. This should be compared with the discussed project of ITER where not before 2015, 500 second long pulses may produce a gain of 0.3 fusion produced electric energy per input electricity $^{[36 a]}$.

The research for the fast ignitor as initiated by Mike Campbell is now a broad stream of research worldwide. The irradiation of the laser on a target usually has a prepulse which is leading to relativistic self-focusing as measured in the numerous experiments since 1975 and fully understood by theory, see above (Fig. 3). The petawatt pulses resulted in relativistic effects never seen before as large amounts of positrons from pair production ${ }^{[37,38]}$ gamma bursts of $10 \mathrm{MeV}$ and higher energy producing nuclear transmutation by nuclear photo effect, ion energies in the $\mathrm{GeV}$ range and electron beams in the $100 \mathrm{MeV}$ range ${ }^{[39]}$ etc.

Discovery of the skin layer acceleration of plasma blocks by the nonlinear force for laser fusion with uncompressed solid DT fuel: Within this broad stream of research there were some very special exceptions with drastic anomalies. There were the measurements of Badziak et al. In 1999 on ion emission by TW-ps laser pulses where instead of the expected $22 \mathrm{MeV}$ $\mathrm{Cu}^{+10}$ from relativistic self-focusing as seen in all similar experiments, the ions had an energy of the half $\mathrm{MeV}$ only. When varying the laser intensity by a factor 30 , their energy changed in the same way, but the ion number was completely unchanged. The explanation ${ }^{[40]}$ was as clear as simple: there was no sufficient prepulse to generate a plasma in front of the target to permit relativistic self-focusing and the interaction occurred only in the (nearly) constant volume of the skin layer, Fig. 6, what was immediately understood in view of the earlier computations, Fig. 5.

This skin layer acceleration of plasma was then confirmed in detailed experiments and computations $^{[41,42]}$. It was helpful to remember the $10^{18}$ $\mathrm{W} / \mathrm{cm}^{2}$ plane geometry computations for the ps duration (PhD. V. Lawrence 1978) where such a skin layer acceleration (SLA) by the nonlinear force within 2 ps was shown. A several wavelength thick deuterium plasma block was moving in a very directed way against the laser and another block into the target. As expected 2002, these nearly space charge neutral blocks had ion current densities up to $10^{11} \mathrm{Amp} / \mathrm{cm}^{2[40,41]}$ as measured then and reproduced by computations in all details, Fig. 7. The fact that the prepulse was sufficiently suppressed (contrast ratio $10^{8}$ until 50 ps before the main pulse) was seen very convincingly in a paper by Zhang et al. ${ }^{[43]}$ ) where the x-ray emission from targets at 100fs-TW Ti: sapphire laser irradiation was measured. These x-rays were of unexpected low intensity in contrast to all the usual experiments (where indeed prepulses permitted relativistic self-focusing the subsequent extreme high intensities necessary for higher x-ray emission). When similar 7\% intense 100fs pules were pre-irradiated at varying times, nothing changed 20 to 50ps precursor time. But at 70ps and longer times, the usual high x-ray emission was seen. The $70 \mathrm{ps}$ prepulse permitted the establishing of a plasma plume necessary for the relativistic selffocusing as usual, Fig. 6.

An experiment by Sauerbrey ${ }^{[45]}$ using Schäfer's TW-0.3ps KrF laser pulse focused to about 30 
wavelength diameter as usual on a target, showed an acceleration of the plasma front by $10^{20} \mathrm{~cm} / \mathrm{s}^{2}$ from Doppler measurement. Exactly this was calculated by nonlinear force acceleration for an undistorted plane plasma front. This confirmed indirectly that there was not sufficient prepulse to produce self-focusing and the ideal plane front geometry conditions were fulfilled. When Sauerbrey tried to repeat this later with TiSapphire laser pulses, no similar Doppler shift was seen. Obviously the prepulse in this case resulted in self-focusing and destroyed the plane geometry interaction.

The measured low temperature plasma blocks of about $30 \mu \mathrm{m}$ diameter width, $10^{11} \mathrm{~A} / \mathrm{cm}^{2}$ DT ion current densities with resonance-adjusted $80 \mathrm{keV}$ energy could be considered for ignition of uncompressed solid DT. Similar low compression large Amount DT was considered by Nuckolls and Wood ${ }^{[46]}$ to be ignited by electron beams as a fast ignition experiment. In our 80 $\mathrm{keV}$ DT block ignition, a $10 \mathrm{~kJ}$ laser pulse could well produce $100 \mathrm{MJ}$ fusion energy in a fully controlled way ${ }^{[44]}$ similar to the before mentioned case with electron beams but avoiding any complication of precompression. A necessary condition for the declassified $^{[47]}$ SLA-nonlinear force block ignition is that the laser pulse energy is high enough. The Badziak et al. experiments are close to $10^{6} \mathrm{~J} / \mathrm{cm}^{2}$. The necessary condition of $4 \times 10^{8} \mathrm{~J} / \mathrm{cm}^{2}$ (Bobin 1971) should be available with shorter laser wavelengths while not destroying the other conditions. Whether this may lead to a new modified direction of Campbell's fast ignitor for a very simplified fusion power reactor is just under discussion.

\section{REFERENCES}

1. Working under the supervision of Paul Görlich, the $\mathrm{R}$ and $\mathrm{D}$ director of Zeiss in Jena, I clarified the long years controversy about the thermionic and photoelectric Richardson equations for electron emission [P. Görlich, H. Hora, W. Macke, Experim. Techn. der Physik 5, 217 (1957)] from solids which I finally developed with the result, that for temperatures above $\mathrm{mc}^{2}$, electrons have no Fermi statics [Zeitschr. f. Physik 164, 359 (1961)] breaking the result of Pauli of 1955, that particles can be only fermions or bosons, see S. Eliezer, A. Ghatak, H. Hora, E. Teller, Introduction to Equations of State, Cambridge Univ. Press 1986, Chapter 1. -- To Görlich's fundamental discovery of the $\mathrm{Cs}_{3} \mathrm{Sb}$ photocathode of 1936 , I clarified that these everywhere used photocathodes have a volume photoeffect in contrast to the established theories by Fröhlich, Tamm R.W. Pohl etc. And proved my concluded anomalous vectoreffect experimentally. [H. Hora, Annalen der Physik, 10,
243 (1963)] - My familiarity with this semiconductor and later with the multi-Valey-band silicon and the direct-band GaAs (after working with Gert Folberth at IBM-Germany) enabled me to predict the laser-threshold of these materials under electron beam excitation. This was presented April 1964 at the Siemens Research Lab Munich and fully agreed with later published first experiments. My presentation at the International Laser Conference Bern, September 1964 was especially highlighted by the Neue Züricher Zeitung [H. Hora, Zeitschrift f. Naturforschung 20A, 543 (1965)].

2. Hora, H., 1965. Institute of Plasma Physics Garching Report 6/23 (Juli 1964); National Research Council of Canada, technical translation TT-1193.

3. Hora, H., 1991. Plasmas at High Temperature and Density (Springer, Heidelberg 1991).

4. Teller, E., 1972. IEEE J. Quantum Electronics 8.

5. Nuckolls, J.H., H. Schwarz and H. Hora Eds., 1973. LIRPP (Laser Interaction and Related Plasma Phenomena) 3B: 399.

6. Isenor, R.N., 1964. Appl. Phys. Lett. 4, pp: 152.

7. Linlor, W.I., 1963. Appl. Phys. Lett. 3, pp: 210.

8. Hora, H., D. Pfirsch, A. Schlüter, Zeitschr and Naturforsch, 1976. 22A: 278.

9. Hora, H, 1969. Phys. Fluids, 12: 182.

10. Hora, H. 2000 Laser Plasma Physics: Forces and the Nopnlinearity principle (SPIUE Press, Bellingham, WA).

11. Boreham, B. W. and H. Hora, 1974. Phys. Rev. Lett. 42, pp: 776.

12. Cicchitelli, L., H. Hora and R. Postle, 1990. Phys. Rev. A4, pp: 3727.

13. Hora, H., 1995. Elektrodynamik (S. Roderer, Regensburg 1995).

14. Klima, R., V. Petrzilka and J. Cz., 1972. Physics B22, pp: 896.

15. Hora, H., 1985. Phys. Fluids 28, pp: 3706.

16. Hora, H. and f. Zeitschr, 1969. Physik 226, pp: 156.

17. Hora, H., 1975. J. Opt. Soc. Am. 65, pp: 882.

18. Jones, D.A., E.L. Kane, P. Lalousis, P.R. Wiles and H. Hora, 1982. Phys. Fluids 25, pp: 2995.

19. Hauser, T., W. Scheid and H. Hora, 1992. Phys. Rev. A45, pp: 1278.

20. Chen, F.F., LIRPP 3A, pp: 291.

21. Gabor, D., 1953. Prof. Roy. Soc. LondonA213, pp: 73.

22. Hora, H. P.S. Ray and Z. Naturforsch, 1978. A33: 890.

23. Kirkpatrick, R. and J.A. Wheeler, 1981.Nuclear Fusion 21,pp: 389.

24. Lackner, K.S., S.A. Colgate et al., LIRPP 11, pp: 356. 
25. Hora, H., H. Azechi, Y. Kitagawa et al., 1998. J. Plasma Phys. 60, pp: 743.

26. Hora, H. and M. Aydin, 1992. Phys. Rev. A45, pp: 6123.

27. Maddever, R.A.M., B. Luther-Davies et al., 1990. Phys. Rev. A41, pp: 2154.

28. Hora, H. and M. Aydin, 1999. Laser and Particle Beams17, pp: 209; Osman, F., et al . Am. J. Appl. Sci. 1,76

29. Azechi, H. et al., 1991. Laser and Particle Beams, 9: 193.

30. Hora, H., P. Laousis and S. Eliezer, 1984. Phys. Rev. Lett. 53, pp: 1650.

31. Goldsworthy, M.P., H. Hora and R.J. Stening, 1990.Laser and Particle Beams 8, pp: 33.

32. Hora, H. and A.J. Ghatak, 1985. Phys. Rev. A31, pp: 3473.

33. Hora, H., Gu Min, S. Eliezer, P. Lalousis, R.S. Pease and H. Szichman, 1989. IEEE Trans. Plasma Sc. PS-17, pp: 284.

34. Hora, H. et al., SPIE Proceed. 5448, p: 1190; Hora et al.2005 Astophys. \& Space Sci. 298, 247

35. Campbell, E.M., 2000. Light and Shade, Festschrift at awarding Nobility of Chiyoe Yamanaka, pp: 470.

36. Hora, H., G.H. Miley and F. Osman, 2003. J. Plasma Physics 69, pp: 413.

36a.Hora, H., 2004, Laser and Particle Beams, 22, 439
37. Hora, H., 1973. Nature (Phys. Sc.) 243, pp: 34.

38. Key, M.E., 2001. Nature 412, pp: 775.

39. Hora, H., M. Hoelss, W. Scheid, J.X. Wang, Y.K. Ho, F. Osman and R. Castillo, 2000. Laser and Particle Beams, 18: 135-144.

40. Hora, H., J. Badziak et al., 2002 Opt. Commun., pp: 207-333.

41. Badziak, J., S. Glowacz, S. Jablonski and H. Hora, 2004. Appl. Phys. Lett. 85, pp: 3041.

42. Badziak, J., S. Glowacz, S. Jablonski, P. Parys, J. Wolowski, H. Hora, J. Kraska, L. Laska and K. Rohlena, 2004. Plasma Physics and Controlled Fusion 46, pp: B541.

43. Zhang, P. et al., 1998. Phys. rev. E57, pp: 3746.

44. Miley, G.H., F. Osman, H. Hora, J. Badziak, K. Rohlena, K. Jungwirth, J. Wolowski, Y. Cang, X. He', J. Zhang and P. Hammerling, 2004. SPIE Proceed. 5448, pp: 973.

45. Sauerbrey, R., 1996. Phys. Plasma 3, pp: 4712.

46. Nuckolls, J.L. and LL.. Woods, 2002. Future of Inertial Fusion Energy, Lawrence Livermore National Laboratory, Preprints UCRL-JC-249860 Sept.4.

47. Hora, H., German Patent Appl. 103308515.3 (28.2.2002, declassified 5.9.2002). 